\title{
PROGRESS ON A NEW EXPERIMENTAL TEST OF THE GRAVITATIONAL INVERSE-SQUARE LAW
}

\author{
R.M. BONICALZI, P.E. BOYNTON, M.W. MOORE \\ Physics Department, University of Washington \\ Seattle, WA 98195, USA \\ R.D. NEWMAN, E.C. BERG \\ Physics Department, University of California at Irvine \\ Irvine, CA 92697, USA
}

\begin{abstract}
Progress is reported from an oscillating torsion-pendulum experiment searching for gravitational inverse square law violation (ISLV) between macroscopic bodies with a separation of order $10 \mathrm{~cm}$. An important design feature is the special mass configurations of both the pendulum and gravitational source. These provide high sensitivity to the horizontal derivative of the Laplacian of the gravitational potential, a unique signature of ISLV, while strongly suppressing couplings through Newtonian gravity that can mimic this signature.
\end{abstract}

The Standard Model of fundamental interactions, with General Relativity inelegantly tacked on, is consistent with every observational test to date. Within this vacuum of contradictory evidence, theoretical attempts to unify the laws of nature into a single overarching framework have thrived. We are a collaboration between groups at the University of Washington and the University of California Irvine that conducts table-top torsion-pendulum tests of fundamental aspects of gravity, searching for "new physics". This paper reports progress on one of our experiments.

In a terrestrial lab, the gravitational context is the weak-field limit of General Relativity, i.e. Newton's Law of Universal Gravitation. The experiment reported here is designed to be sensitive to violation of the Newtonian inverse-square law. A mathematically equivalent description, which is useful in making the experiment's design transparent, is the experiment's sensitivity to a non-vanishing Laplacian of the potential describing this two-body interaction.

Many proposed "new physics" interactions violate the inverse-square law. A typical example is the introduction of an ultra-light mediating boson by the addition of a Yukawa term to the standard Newtonian potential. That is, the gravitational potential energy for a pair of point masses is given by

$$
U=-\frac{G m_{1} m_{2}}{r}\left(1+\alpha e^{-r / \lambda}\right),
$$

where $\alpha$ and $\lambda$ parameterize the strength (relative to Newtonian gravity) and characteristic range, respectively, of the Yukawa interaction. This is the model we employ to quantify a putative non-Newtonian interaction in this search for inverse-square-law violation (ISLV). Ultimately, the particular model is not important unless a significant signal were 
detected, then the form of the interaction would be dictated by the data. The current constraints on ISLV in $\alpha-\lambda$ parameter space along with this experiment's design goal is plotted in Figure 1 [1]. At the most sensitive range, $\lambda=12 \mathrm{~cm}$, the goal is to place an upper bound of $10^{-5}$ on $\alpha$, more than an order-of-magnitude improvement.

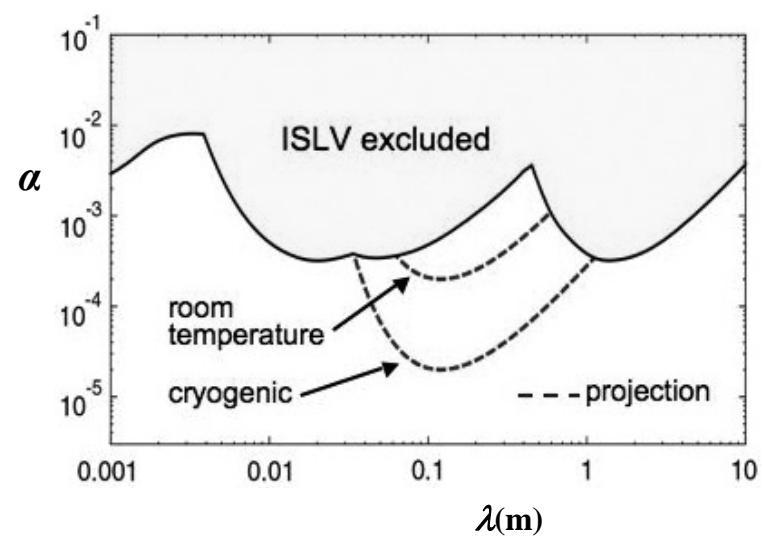

Figure 1. Current $2 \sigma$ empirical bounds on ISLV for positive $\alpha$, with the projected $2 \sigma$ goals of this experimental program.

This experiment examines the interaction (coupling approximately to mass) between two intricately shaped objects: a fist-size pendulum mass composed of fused silica and coated with gold, and a human-scale "source mass" constructed from 1.4 tonnes of precisely machined, stainless-steel cylinders. To isolate the effect of the source from the ambient environment, the source mass rotates through a sequence of static azimuthal positions around the pendulum, which hangs at its center. For separations of order 10 $\mathrm{cm}$, electromagnetism and gravity are the only relevant forces acting. The effects of both are suppressed below the level of ISLV measurement noise to establish a null experiment. Electromagnetic effects are mitigated by common techniques of magnetic and electrostatic shielding. The influence of Newtonian gravity is suppressed by the special geometries of the pendulum and source mass, which are shown and discussed in [2].

The key to this design is a generalization of the standard multipole formalism that provides a clean separation between contributions from Newtonian gravity and possible non-Newtonian (i.e. ISLV) interactions [2]. The mass configurations of both the pendulum and source mass are chosen to satisfy two design goals: 1 ) to exaggerate the lowest-order, torque-producing, non-Newtonian multipole term in the interaction energy

$$
\frac{\partial}{\partial x}\left(\nabla^{2} V_{s m}\right) \int_{p e n d} \rho_{\text {pend }} r^{3} Y_{11} d x d y d z
$$


which explicitly reveals an ISLV character through its proportionality to the horizontal gradient of the Laplacian of the source-mass potential; 2) to suppress the relevant Newtonian terms that could mimic an ISLV signal, by assuring that significant gravitational systematics arise only at second order in pendulum and source-mass fabrication errors.

Our project's most sensitive ISLV search mode involves taking data at cryogenic temperatures. However, an initial operation phase at ambient temperature is providing proof of the design principles and avoids the complication and expense of maintaining a liquid helium bath. Here we present data from this preliminary lower-sensitivity phase.

If the torque observable were chosen to be either pendulum deflection or oscillation frequency, sensitivity to torsion-fiber-temperature drifts and corresponding changes in the fiber's torsion constant would become problematic. However, we have used the amplitude of the second-harmonic distortion of the fundamental oscillatory mode as our torque observable. Due to its relative insensitivity to thermal effects, no heroic measures need be taken to ensure extreme temperature stability [3]. At each source-mass position, the pendulum is set into large-amplitude torsion oscillation. Using an optical lever, the detailed motion of the pendulum is reconstructed by precisely measuring the times at which it rotates through a set of fiducial azimuth orientations.

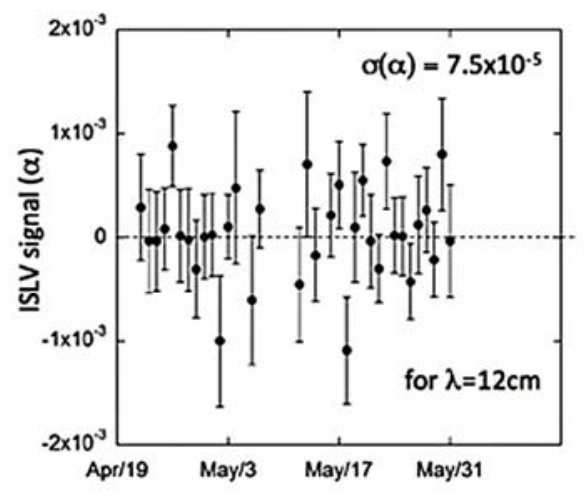

Figure 2. ISLV data from the spring of 2009.

Data from 2009 are shown in Figure 2, where each point represents approximately one day of observation. The vertical axis indicates the value of $\alpha$ implied by the measurement of the second-harmonic amplitude from that run, assuming the most sensitive value of the range, $\lambda=12 \mathrm{~cm}$. These 34 points determine an overall rms uncertainty of $7.5 \times 10^{-5}$ in the measurement of $\alpha$. Because of an instrument-tilt-induced systematic effect discovered just before this conference (not shown in Table 1), we are presently unable able to use these data to set an improved bound on $\alpha$. 
Table 1 lists the limits that have been placed on various systematic effects stated in terms of their contributions to $\alpha$, along with the basic approach to mitigating each effect. For brevity, only the largest three gravitational-multipole contributions are listed. The $l=2$ and $l=3$ terms are measured by utilizing special pendulum and source-mass configurations that exaggerate the relevant mass or field moments. The $l=4$ value is an estimate based on known fabrication-error bounds that are assumed to have spatial distributions that maximize this contribution.

Table 1. Established limits on various systematic effects.

\begin{tabular}{|c|c|c|}
\hline Cause & Remedy & Contribution \\
\hline $\begin{array}{c}\text { Variation in torsion-fiber } \\
\text { temperature }\end{array}$ & $\begin{array}{c}\text { Exploit 2nd-harmonic } \\
\text { temperature insensitivity }\end{array}$ & $\alpha<3 \times 10^{-7}$ \\
\hline $\begin{array}{c}\text { Fiber contribution to tilt } \\
\text { sensitivity }\end{array}$ & $\begin{array}{c}\text { Exploit 2nd-harmonic } \\
\text { tilt insensitivity }\end{array}$ & $\alpha<6 \times 10^{-6}$ \\
\hline Magnetic torques & $\begin{array}{c}\text { 4-layer concentric } \\
\text { magnetic shield }\end{array}$ & $\alpha<1 \times 10^{-7}$ \\
\hline $\begin{array}{c}\text { Temperature gradient in } \\
\text { pendulum environment }\end{array}$ & $\begin{array}{c}\text { 6-layer concentric } \\
\text { thermal shield }\end{array}$ & $\alpha<5 \times 10^{-6}$ \\
\hline \multirow{2}{*}{ Newtonian gravity } & $\begin{array}{c}\text { design of pendulum } \\
\text { and source } \\
\text { mass distribution }\end{array}$ & $\alpha_{21}=(0.0 \pm 0.7) \times 10^{-6}$ \\
\cline { 3 - 4 } & \multicolumn{2}{c}{$\alpha_{31}=(0.8 \pm 1.3) \times 10^{-6}$} \\
\cline { 3 - 4 } & \multicolumn{2}{|c}{$\alpha_{41}= \pm 1.8 \times 10^{-6}$} \\
\hline
\end{tabular}

Progress continues as we work to understand and reduce the recently identified tilt bias, while also preparing for cryogenic operation to begin in the spring of 2010.

\section{Acknowledgments}

This work is supported by the US National Science Foundation under grants PHY0701923 and PHY-0701707, by the Pacific Northwest National Laboratory, and by the Boeing Company.

\section{References}

1. R.D. Newman, E.C. Berg and P.E. Boynton, Space Sci. Rev. 04 June (2009).

2. P.E. Boynton, R.M. Bonicalzi, A.M. Kalet, K.J. McKenney, M.W. Moore, J.H. Steffen, E.C. Berg, W.D. Cross, R.D. Newman, R.E. Gephart (2007), “Gravitation Physics at BGPL”, New Astronomy Reviews, 51, pp 334-340

3. Moore M W and Boynton P E (1996) Proc. 31st Rencontres de Moriond, pp 423-428 